\title{
Acute effects of physical exercise with microcurrent in the adipose tissue of the abdominal region: A randomized controlled trial
}

\author{
Andreia Noites, $\mathrm{PhD}^{\mathrm{a}, *}$, Anabela Moreira, BSc ${ }^{\mathrm{b}}$, Cristina Melo, $\mathrm{PhD}^{\mathrm{a}}$, Miriam Faria, $\mathrm{PT}^{\mathrm{a}}$, \\ Rui Vilarinho, MSc ${ }^{\mathrm{a}}$, Carla Freitas, PT ${ }^{\mathrm{a}}$, Pedro R.R. Monteiro, PhD ${ }^{\mathrm{c}}$, Paulo Carvalho, PhD ${ }^{\mathrm{a}}$, \\ Nuno Adubeiro, MSc ${ }^{\mathrm{d}}$, Manuela Amorim, MSc ${ }^{\mathrm{b}}$, Luísa Nogueira, PhD ${ }^{\mathrm{d}}$, Rubim Santos, PhD \\ a Department of Physiotherapy, Activity and Human Movement Study Center, School of Allied Health Technologies-Polytechnic Institute of Porto, Portugal \\ ${ }^{\mathrm{b}}$ Department of Clinical Analysis and Public Health, School of Allied Health Technologies-Polytechnic Institute of Porto, Portugal \\ ${ }^{\mathrm{c}}$ Department of Physiology, Activity and Human Movement Study Center, School of Allied Health Technologies-Polytechnic Institute of Porto, Portugal \\ d Department of Radiology, School of Allied Health Technologies-Polytechnic Institute of Porto, Portugal \\ e Department of Physical and Activity and Human Movement Study Center, School of Allied Health Technologies-Polytechnic Institute of Porto, Portugal
}

A B S T R A C T

Keywords:

Abdominal fat

Microcurrent

Aerobic exercise

Adipose tissue

Electrical stimulation

Fat cell lipolysis

Randomised controlled trial
Introduction: Increased abdominal fat and sedentary lifestyles contribute to cardiovascular disease risk. Low-intensity electrical current (microcurrent) on the abdominal region, associated with physical exercise, appears to be an innovative method to increase the lipolytic rate of abdominal adipocytes, in order to reduce abdominal fat. This study aimed to analyze the acute effects of microcurrent associated with an aerobic exercise program in healthy subjects in lipolysis.

Method: A double-blinded, randomized controlled trial was developed and conducted in a higher education school. Eighty-three healthy subjects, aged between 18 and 30 years old and with a 18.5 to $29.9 \mathrm{~kg} / \mathrm{m}^{2}$ body mass index were randomly assigned either to an experimental or to a placebo group. Subjects received a trans-abdominal microcurrent stimulation for 40 min with (experimental group) or without (placebo group) electrical current, followed by a single aerobic exercise session (60 min at $45-$ 55\% VO2max intensity). Lipolytic activity (serum glycerol), abdominal fat (waist circumference, abdominal skinfold, ultrasonography), and serum lipid profile (serum triglyceride, total cholesterol, lowdensity lipoprotein cholesterol and high-density lipoprotein cholesterol) were evaluated in all subjects. Physical activity (International Physical Activity Questionnaire) and dietary intake (food-frequency questionnaire) questionnaires were applied.

Results: After the intervention, lipolytic rate was significantly higher $(\mathrm{p}=0.003)$ in the experimental group (mean $=0.15$ ) than in the placebo group (mean=0.09). Glycerol results showed a statistically significant increase between baseline and after the intervention for both experimental group $(p=0.001)$ and the placebo group $(\mathrm{p}=0.001)$.

Conclusion: Combined use of microcurrent and physical aerobic exercise had an acute effect enhancing lipolytic rate comparing to exercise alone, in young healthy subjects.

\section{Introduction}

Abdominal obesity, namely visceral and deep subcutaneous adipose tissue, are associated with a greater risk for the development of metabolic and cardiovascular complications than in other regions of the body [1-3]. These tissues show higher metabolic (pro-inflammatory, lipogenic, and lipolytic) activity and subsequently higher proportion of saturated fatty acids [4,5]. This enables an increasing in blood triglycerides, which may culminate in dyslipidemia, diabetes, hypertension and, thus, increased cardiovascular disease risk $[3,6]$. However it has been widely discussed in the literature, that not only individuals with a body mass index (BMI) considered high or excessive can present a dysfunction in adipocyte behavior, but also it can be found in adipocytes in individuals with normal BMI [7].

Among others, age, gender, genetics, hormones and ethnicity are broad etiological factors that contribute to a variation in adipose tissue accumulation and plasma lipids [8,9]. A hypercaloric and fat-rich diet, together with sedentary lifestyle, also play a 
major role in the development of abdominal obesity, thus lifestyle modification programmes regarding nutrition, physical activity and weight control/loss are a major public health concern nowadays, showing promising results $[10,11]$.

Moderate aerobic exercise increases blood flow and enables fatty acid delivery from the adipose tissue to skeletal muscles during muscular activity $[12,13]$. Catecholamines (adrenaline and noradrenaline) regulate lipolysis during exercise, stimulating and inhibiting lipolysis through beta-adrenergic and alpha-adrenergic pathways respectively, and thus increasing fatty acids and glycerol secretion from the adipose tissue [12,14]. In addition, exercise promotes muscular cytokines secretion, which may induce systemic effects in the adipose tissue through IL-6 mediated lipolysis and fatty acids oxidation, and irisin-induced white adipose tissue to brown adipose tissue turnover [15]. Also, the use of transcutaneous microcurrent together with aerobic exercise appears to have a cumulative effect in the mobilization of the abdominal adipose tissue, providing means to local abdominal fat treatment [16].

A microcurrent is characterized by the application of a low frequency and low intensity current, which stimulates triglycerides hydrolysis, hence releasing free fatty acids and glycerol into the blood circulation [17]. Fatty acids become then available for oxidation and energy expenditure during physical exercise [18-21]. On the other hand, venous glycerol can indicate lipolytic activity in adipose tissue, because is not reutilized for the resynthesis of new triglycerides due to unavailability of glycerol kinase in adipose tissue [7,14].

The present study aims to assess the effect of a single application of abdominal microcurrent, in addition to a single session of aerobic exercise, in acute lipolytic activity, as evaluated by the serum glycerol levels variation in clinically healthy individuals. It was aimed, also, to evaluate if the gender and the amount of adipose tissue are related to the effects of microcurrent on the lipolytic activity.

\section{Methods}

\subsection{Study design, randomization, and implementation}

This study was a double-blinded, randomized controlled trial (RCT) and performed between January 2013 and March 2014.

The study was approved by the Ethics Board (nr. 0325/2013 and nr. 04131/2013) and Direction $\left(n^{\circ}\right.$ 000276) at the central coordinating and participating institution. The trial was registered at the US National Institutes of Health (ClinicalTrials.gov) \#NCT02110927. All participants provided informed consent in compliance with the principles of the Declaration of Helsinki.

Following eligibility screening by the research coordinator, eligible subjects were randomly allocated by sex into the placebo and experimental groups. The randomization schedule was stratified in mutable blocks of 4 , through a unique random number designation (Microsoft Office Excel). Apart from the researcher responsible for the electrolipolysis protocol, all other researchers and participants were blinded to the intervention allocation.

\section{Participants}

The study was developed and conducted in a higher education school. All students $(n=1966)$ were invited to participate by electronic email and answer a sample characterization and selection questionnaire. We assessed 196 participants who volunteered responding to an email, aged between 18 and 30 years old and with a 18.5 to $29.9 \mathrm{~kg} / \mathrm{m}^{2}$ BMI. Students were excluded according to the following exclusion criteria: pregnant, pregnant in the previous year or planning to get pregnant soon; clinical history of cancer; metabolic, renal or digestive dysfunctions; abdominal surgery; contraindications to microcurrent application, such as abdominal, dermatologic or tactile sensibility dysfunctions; pacemakers; intrauterine contraceptive device; osteosynthesis material in the abdominal region; conditions limiting aerobic exercise such as cardiovascular, respiratory or orthopaedic diseases; involvement in other fat reduction procedures/programmes; and hypocaloric diets. A total of 101 volunteers were excluded and the 95 subjects eligible to participate in the study were randomly assigned to the experimental group (EG) $(n=47)$ or to the placebo group (PG) $(n=48)$ (Figure I). Excel softwear was used to randomize participants into the groups. Five subjects in the EG and seven subjects were lost in the PG (Fig. 1). Thus, at the end of the study, the EG data was available for 42 subjects ( 16 males; 26 females) and for PG, 41 subjects ( 15 males; 26 females)

\subsection{Measurements}

\subsubsection{Anthropometrics}

Height was assessed by a standard, wall mounted stadiometer, before intervention. Body mass and fat mass percentage were measured by bio-impedance using a Tanita Inner Scan BC-522 (Tanita, Tokyo, Japan), with the subjects wearing light clothing without shoes [22]. For the bio-impedance evaluation, the volunteers were instructed not to drink alcohol nor to perform vigorous physical exercise before $24 \mathrm{~h}$, as well as to avoid heavy meals and to empty the bladder before the measurement.

The BMI was calculated according to the World Health Organization guidelines [23].

\subsection{Abdominal fat}

Waist circumference was measured at the midpoint between the lowest rib and the iliac crest.

Abdominal skinfold and suprailiac skinfold were measured using an analogic Caliper Harpenden (Baty International, West Sussex, United Kingdom) [24].

Ultrasonography was performed using a ViamoTM ${ }^{7}$ ultrasound system (Toshiba Medical Systems, Tustin, California) to measure subcutaneous and visceral abdominal fat below the xiphoid apophysis, and navel fat below the navel [16].

All the measurements were evaluated before intervention.

\subsection{Physical activity}

Subjects' physical activity levels were assessed, on the day of the intervention protocol, through the abridged form of the International Physical Activity Questionnaire (IPAQ) application, previously validated, which records the duration of physical activity reported as metabolic equivalent task (MET) minutes/ week, and categorizes the physical activity undertaken in the previous 7 days on three levels (low, moderate, and high) $[25,26]$.

\subsection{Dietary intake}

Semi-quantitative Food Frequency Questionnaire, referring to 12 months prior to the study, was used to monitor dietary intake. All subjects answered to the questionnaire on the day of the intervention protocol. The Food Processor Plus ${ }^{\circledR}$ (ESHA Research, Salem, Oregon) was used to convert food into nutrient (consumption of caffeine, sugar, calcium, magnesium, alcohol, protein, carbohydrates, total fiber, sodium, total fat, saturated fat, monounsaturated fat, cholesterol, $n-3$ and n-6 fatty acids, and total energy intake) [27]. 


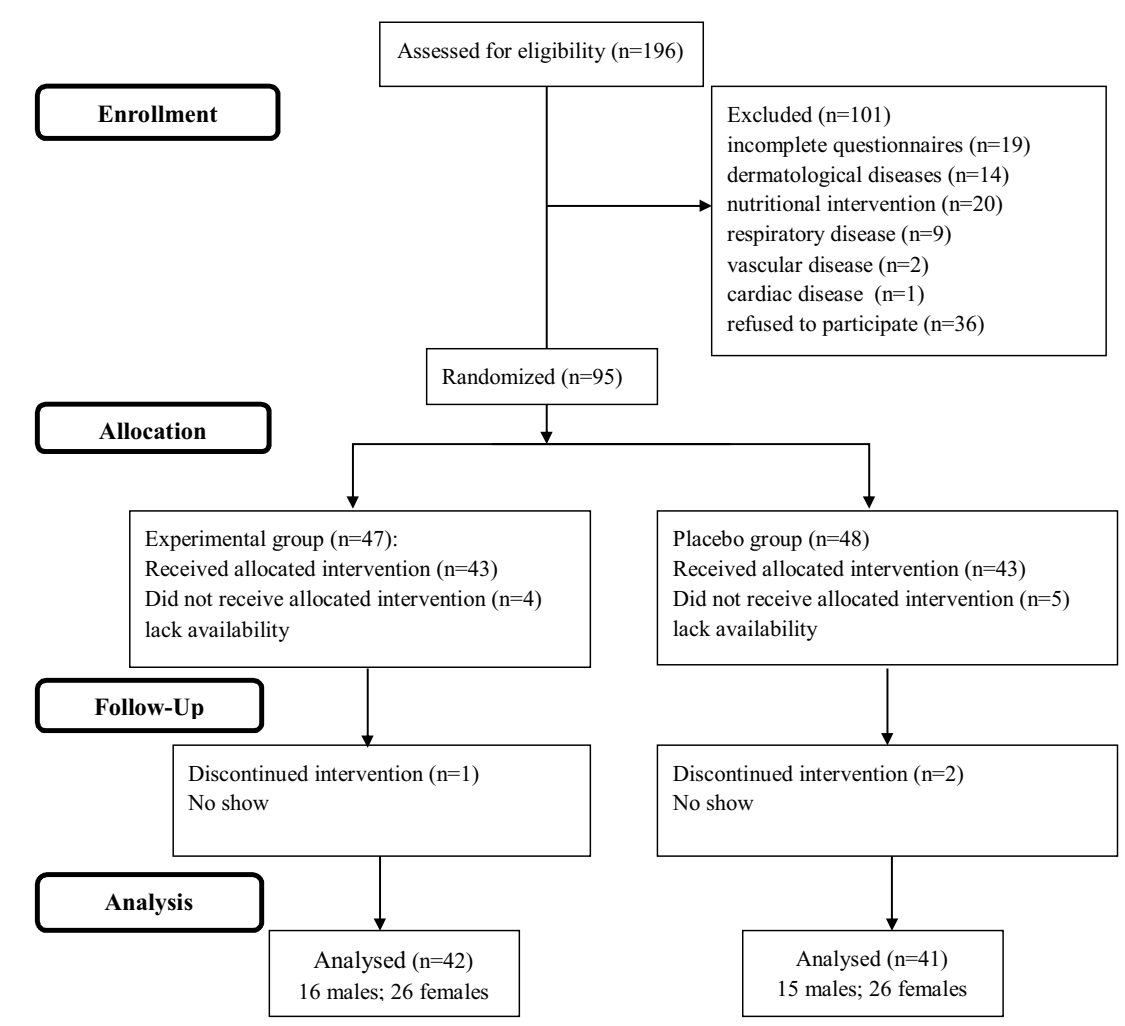

Fig. 1. - Flow diagram of patients.

2.6. Serum glycerol, triglycerides, total cholesterol LDL and HDL cholesterol

Blood sample were collected before and after the intervention. The blood collection after the intervention was made 5 min after aerobic exercise with subject in rest.

Blood samples from all subjects were collected through a standard antecubital venepuncture to $8 \mathrm{ml}$ serum-separator tubes (BD Vacutainer ${ }^{\circledR}$ System, BD Diagnostics, Franklin Lakes, NJ), immediately prior to- Baseline (M0) and after the intervention protocol (M1). Serum was separated by low speed centrifugation (4500 rpm, $10 \mathrm{~min}, 22^{\circ} \mathrm{C}$ ) (Sigma ${ }^{\circledR} 3 \mathrm{k} 15$, SIGMA Laborzentrifugen $\mathrm{GmbH}$, Osterode am Harz, Germany), transferred into aliquots, and stored at $-80^{\circ} \mathrm{C}$ until the analysis. Serum triglyceride, total cholesterol, Low-density lipoprotein (LDL) cholesterol and highdensity lipoprotein (HDL) cholesterol (P.Z. Cormay, Lublin, Poland) and glycerol (Randox Laboratories, Crumlim, United Kingdom) concentrations were determined by a Prestige $24 \mathrm{i}$ autoanalyzer ( $\mathrm{P}$. Z. Cormay, Lublin, Poland) using standard enzymatic colorimetric techniques.

\section{Interventions}

Subjects performed one microcurrent session with (experimental group) or without (placebo group) electrical current, followed by a single aerobic exercise session (both groups). Blood samples from all subjects were collected before and after the intervention.

\subsection{Microcurrent protocol}

The non-invasive microcurrent protocol, applied before physical exercise, consisted in the application of low frequency bipolar square-wave, alternated with electrical current to the lower abdominal region using transcutaneous band electrodes
$(25 \times 5,5 \mathrm{~cm} ; 30,5 \times 5,5 \mathrm{~cm} ; 35 \times 5,5 \mathrm{~cm})$ on conductive gel, with a distance of $5-10 \mathrm{~cm}$ between them. A $25 \mathrm{~Hz}$ frequency (impulse duration and resting duration of $20 \mathrm{~ms}$ ) was used during the first $20 \mathrm{~min}$, followed by another application of $20 \mathrm{~min}$ at $10 \mathrm{~Hz}$ frequency (impulse duration and resting duration of $50 \mathrm{~ms}$ ). In the experimental group, the microcurrent's intensity was under each individual's sensitivity threshold (registered values were superior to $700 \mu \mathrm{A}$ and inferior to $1000 \mu \mathrm{A}$ ) [16,28,29]. In the placebo group, all the procedures were similar to the EG but the microcuurent device was off.

In fact the most studied microcurrent protocols, use frequencies of $10 \mathrm{~Hz}$ and $25 \mathrm{~Hz}$ in order to potentiate the effect of microcurrent in local blood flow stimulation and through stimulation of lipolytic mediators released by the sympatic adrenergic system. [16,28,29].

There were no harmful or adverse events during the study. As only mild electrical current was used, no perceptible muscle contraction or muscle pain was generated in the subjects.

\subsection{Exercise protocol}

Aerobic exercise of moderate intensity at $45-55 \% \mathrm{VO}_{2} \max$ (Karvonen's formula) was performed by both groups on a cycloergometer for $50 \mathrm{~min}$, right after the microcurrent protocol, being assessed by the Borg's scale (grade: 12-13), together with a Polar ${ }^{\mathbb{R}}$ heart monitor used to control heart rate [30,31].

Fatty acids oxidation increases progressively during aerobic exercise with moderate intensity, with intensities between 45 and $55 \% \mathrm{VO}_{2} \max [21,32]$.

\subsection{Sample size calculation and statistical analysis}

The power calculation was computed a priori based on data obtained from a pilot study (15 individuals divided by groups received the protocol treatment, since there is no effect size value in the literature). The sample size for the present study was 
calculated to ensure statistical power for a two-group analysis with the variable difference (M1-M0) of glycerol, assuming a power of $90 \%$ and $5 \%$ significance level, revealing a need for 40 patients to detect an effect size of 0.74 .

Baseline characteristics of the participants in both placebo and experimental groups were reported using frequency distributions and descriptive statistics, including measures of central tendency (mean or median) and dispersion (standard deviation or interquartile deviation).

Measured variables were compared between groups (at M0, M1 and M1-M0 difference) and between moments (M0 vs M1), within each group, using an independent two-sample $t$-test and paired samples $t$-test respectively, when normal distribution was assured through the Shapiro-Wilk test. When normality was not verified, the Mann-Whitney test was used for between-group comparisons and the Wilcoxon test for within-group comparisons.

Pearson's correlation coefficient was applied to assess correlation between glycerol mobilization and anthropometric measures, BMI values, physical activity values, abdominal fat, serum triglycerides and serum cholesterol.

Statistical analysis were performed using a Statistical Package for the Social Sciences (SPSS), version 23 (IBM), with a significance level of $5 \%(p<0.05)$.

\section{Results}

A total of 83 randomized subjects completed the trial: 42 subjects in the experimental group (16 males; 26 females) and 41 subjects in the placebo group (15 males; 26 females).

The mean age of the total participants was $20 \pm 1.53$ years, and mean BMI was $22.86 \pm 2.97$ (80\% of the total participants presented normal BMI, while $20 \%$ had an overweight BMI) (WHO, 2014) with no statistically significant differences between groups (Table 1 ).

Baseline (M0) anthropometric characteristics (muscle mass, waist circumference, waist-height ratio and skinfold) and physical activity (total MET's for week) were similar in both groups, with no statistically significant differences (Table 1 ).

In abdominal fat measured by ultrasonography, subcutaneous and visceral waist FAT were similar in PG and EG at baseline (M0) without any significant differences (Table 2).

Table 1

- Baseline demographic and anthropometric characteristics.

\begin{tabular}{llll}
\hline & Mean (SD) & & \\
\hline & EG & PG & $p$ EG vs. PG \\
Age (years) & 20.1 & 20.2 & 0.661 \\
& $(1.5)$ & $(1.5)$ & \\
Height (m) & 1.69 & 1.70 & 0.513 \\
& $(0.1)$ & $(0.1)$ & \\
Body mass (kg) & 65.9 & 65.3 & 0.791 \\
& $(11.8)$ & $(10.8)$ & \\
BMI (kg/m $\left.{ }^{2}\right)$ & 23.2 & 22.5 & 0.322 \\
& $(3.3)$ & $(2.6)$ & \\
Muscle Mass (kg) & 50.6 & 49.6 & 0.815 \\
Waist circumference (cm) & $(12.2)$ & $(10.4)$ & \\
& 79.7 & 79.3 & 0.798 \\
Waist-height ratio & $(7.7)$ & $(7.1)$ & \\
& 0.47 & 0.46 & 0.445 \\
Abdominal skinfold & $(0.03)$ & $(0.03)$ & \\
(mm) & 13.6 & 12.5 & 0.211 \\
Suprailiac skinfold (mm) & $(4.3)$ & $(3.8)$ & \\
IPAQ (MET-min/week) & 14.7 & 15.6 & 0.531 \\
& $(5.7)$ & $(6.2)$ & \\
& 2356.4 & 2010.1 & 0.142 \\
& $(1394.3)$ & $(1099.5)$ & \\
\hline
\end{tabular}

Abbreviations BMI. Body Mass Index; EG. experimental group; PG placebo group; $I P A Q$. international physical activity questionnaire.

$p$ value for initial moment with $t$-test.
Regarding dietary intake, food-frequency questionnaire results showed no statistically significant differences between the experimental group and the placebo group at baseline (M0) (Table 3).

Baseline (M0) lipid and glycerol serum values were similar in both groups.

Serum glycerol results showed a statistically significant increase between M0 and M1 (after the intervention), both in the EG ( $p=0.001)$ and the PG $(p=0.001)$ (Table 4). At M1, glycerol levels in the EG were significantly higher than in the PG $(p=0.003)$, as well as regarding the difference variable $(\mathrm{M} 1-\mathrm{M} 0)(\mathrm{p}=0.004)$. (Table IV).

The lipids profile (total cholesterol, TG, LDL and HDL) results between groups showed no statistically significant differences in M1 and in the difference variable. However, the results in PG showed a statistically significant increase in total cholesterol $(\mathrm{p}=0.001)$, triglycerides $(\mathrm{p}=0.023)$ and LDL $(\mathrm{p}=0.005)$ between M0 and M1. (Table 4).

Regarding the glycerol difference (M1-M0) and total body fat, we observed a moderate and significantly positive correlation in both groups (Table 5). On the other hand, for the visceral and subcutaneous fat measured by ultrasound, as well as for the abdominal circumference and skin folds, a significant correlation between glycerol mobilization and these variables was only noticeable in the placebo group (Table 5). No significant correlations were observed regarding triglycerides, cholesterol, BMI and IPAQ results in both groups (Table 5).

In the gender-related analysis of glycerol mobilization (Fig. 2), at M1 women of both experimental and placebo groups mobilized more glycerol than men $(\mathrm{p}=0.014$ and $\mathrm{p}=0.024$, respectively). Both genders showed higher glycerol levels after the intervention in the experimental group, in comparison with the placebo group $(\mathrm{p}=0.030$ and $\mathrm{p}=0.004$, respectively).

\section{Discussion}

The groups showed similar characteristics in variables age, anthropometric measures, level of physical activity and dietary intake, which reforce the comparison between groups. This information ensures control of the factors that influence metabolism [33].

We used serum glycerol levels as a lipolysis marker, as a measurement of triglycerides hydrolysis because, after lipolysis, glycerol enters into the bloodstream and is not significantly reutilized into triglycerides synthesis due to low levels of glycerol kinase in the adipose tissue [7], while free fatty acids (FFA) may be oxidised during moderate exercise of long duration [34,35]. Although glycerol is mostly metabolized in the liver to later rejoin the glycolysis and gluconeogenesis pathway as glyceraldehyde 3-phosphate- GA3P, a small part may be used in muscles to oxidation and triglycerides synthesis [14,34]

Table 2

- Baseline abdominal fat measured by ultrasonography.

\begin{tabular}{llll}
\hline & Mean (SD) & Dif. Groups \\
\hline & EG & PG & $p$ EG vs PG \\
Subcutaneous Fat - waist $(\mathrm{mm})$ & 14.41 & 14.39 & 0.994 \\
& $(9.03)$ & $(8.79)$ & \\
Visceral Fat - waist $(\mathrm{mm})$ & 5.54 & 5.58 & 0.963 \\
Total Fat iliac crest $(\mathrm{mm})$ & $(3.49)$ & $4.62)$ & \\
& 21.80 & 24.91 & 0.390 \\
\hline
\end{tabular}

Abbreviations EG. experimental group; $P G$ placebo group.

$p$ value for initial moment with $t$-test. 
Table 3

Characterization of dietary intake, by group.

\begin{tabular}{|c|c|c|c|}
\hline & Md (IQR) & & Dif. Groups \\
\hline & EG & PG & $p$ EG vs PG \\
\hline $\begin{array}{r}\text { Calories } \\
(\text { Kcal })\end{array}$ & 2208.37 (376.21) & 2699.19 (812.49) & 0.297 \\
\hline $\begin{array}{l}\text { Carbohydrates } \\
\text { (g) }\end{array}$ & $266.80(61.35)$ & $324.22(101.26)$ & 0.545 \\
\hline $\begin{array}{l}\text { Protein } \\
\quad(\mathrm{g})\end{array}$ & $100.43(14.03)$ & $120.58(32.58)$ & 0.199 \\
\hline $\begin{array}{l}\text { Total Fat } \\
(\mathrm{g})\end{array}$ & $81.50(14.20)$ & $89.88(36.78)$ & 0.287 \\
\hline $\begin{array}{l}\text { Saturated Fat } \\
(\mathrm{g})\end{array}$ & $25.41(4.61)$ & $27.43(10.73)$ & 0.410 \\
\hline $\begin{array}{l}\text { Monounsaturated Fat } \\
\text { (g) }\end{array}$ & $33.59(7.16)$ & 36.73 (14.07) & 0.335 \\
\hline $\begin{array}{l}\text { Polyunsaturated Fat } \\
\text { (g) }\end{array}$ & $12.92(2.20)$ & $15.53(5.73)$ & 0.276 \\
\hline $\begin{array}{l}\text { Cholesterol } \\
\text { (mg) }\end{array}$ & $293.51(62.61)$ & $364.63(108.06)$ & 0.221 \\
\hline $\begin{array}{l}\text { n-3 Fatty Acid } \\
\text { (g) }\end{array}$ & $1.30(0.41)$ & $1.62(0.53)$ & 0.388 \\
\hline $\begin{array}{l}\text { n-6 Fatty Acid } \\
\text { (g) }\end{array}$ & $9.91(2.53)$ & $10.80(4.53)$ & 0.358 \\
\hline $\begin{array}{l}\text { Total Fiber } \\
\text { (g) }\end{array}$ & $23.23(5.24)$ & $28.58(13.86)$ & 0.171 \\
\hline $\begin{array}{l}\text { Sugars } \\
\text { (g) }\end{array}$ & $121.51(34.83)$ & $134.56(58.75)$ & 0.585 \\
\hline $\begin{array}{l}\text { Alcohol } \\
\text { (g) }\end{array}$ & $1.05(1.32)$ & $1.43(1.32)$ & 0.851 \\
\hline $\begin{array}{l}\text { Calcium } \\
\text { (mg) }\end{array}$ & $1055.05(338.15)$ & $1144.05(386.73)$ & 0.325 \\
\hline $\begin{array}{l}\text { Sodium } \\
\text { (g) }\end{array}$ & $1981.2(394.93)$ & $2467.29(490.41)$ & 0.253 \\
\hline $\begin{array}{l}\text { Caffeine } \\
\text { (mg) }\end{array}$ & $89.10(38.63)$ & $72.51(42.14)$ & 0.325 \\
\hline
\end{tabular}

Abbreviations; EG. experimental group; $P G$ placebo group.

Data are expressed as median (Md) and Interquartile deviation (IQR). $p$ value with Mann-Whitney.

Through the analysis of serum glycerol variation, we observed that a single session of abdominal microcurrent associated to aerobic exercise increased significantly the glycerol mobilization, compared to an isolated aerobic exercise session. According to Ramírez-Ponce et al. (1998, 2002, 2003), adipocytes are sensitive to electric current, presenting voltage-dependent potassium channels [36-38]. Moreover, electrostimulation per se activates lipolytic activity in the human adipose tissue [17]. A recent study showed that mild electric current with heat shock may reduce excessive adipose tissue and metabolic dysfunction [39], hence supporting that the application of abdominal microcurrent may play an significant role in lipolytic activity and sympathetic nervous system stimulation. These results in an increased catecholamine secretion, which in turn activates the adenylyl cyclase pathway leading to the conversion of ATP into cyclic adenosine monophosphate- cAMP and protein kinase A (PKA) activation [14,40,41]. PKA then stimulates hormone-sensitive lipase, which hydrolyses adipocyte-stored triglycerides into free fatty acids and glycerol $[14,40,41]$. In addition, adipocyte triglyceride lipase (ATGL) may be activated simultaneously to hormone-sensitive lipase, resulting in approximately $95 \%$ of triglycerides hydrolysis in adipocytes [40].

As expected, glycerol levels raised between M0 and M1 in the placebo group, which can be explained by the fact that exercise increases energetic demand, promoting catecholamines secretion and, therefore, increasing triglycerides lipolysis rate and blood flow in adipose and muscular tissues, enabling FFA uptake into muscles $[13,42,43]$. On the other hand, the higher levels of glycerol observed in the experimental group show that microcurrent promotes a higher mobilization of abdominal fat, thus enabling higher FFA consumption during moderate aerobic exercise.

As microcurrent application may activate lipolysis, we combined it with a single exercise session in order to promote FFA $\beta$-oxidation and consequently reduce FFA re-esterification in the adipose tissue $[7,44]$. Thus, a longer session (30 min or more) of moderate aerobic exercise $\left(\mathrm{Vo}_{2} \max 25 \%\right.$ to $65 \%$ ) was applied to promote FFA muscle uptake, in opposition to anaerobic exercise, which uptakes preferentially carbohydrates through glycolysis, being more effective on body fat reduction $[13,43]$. Carbohydrates, FFA availability and muscle oxidation may be affected by several factors other than exercise duration and intensity, including dietary intake, physical performance and body composition $[33,43,45]$. Even though we verified the homogeneity of these baseline variables between experimental and control

The increase in triglyceride, total cholesterol and LDL cholesterol observed in the placebo group after exercise may be due to a blood volume decrease [46]. Despite the significance of these results, the clinical relevance of median values is limited.

The lipoprotein values correlation with the amount of glycerol released into bloodstream showed a non-significant and weak correlation in both groups, which can be explained by the fact that glycerol mobilization is not associated with TG and cholesterol levels. However, participants in this sample were healthy individuals, normolipidic and non-obese.

Regarding anthropometric measures, only the placebo group presented a positively significant correlation between the amount

Table 4

Lipids profile and serum glycerol: Cholesterol, Triglycerides, Glycerol, LDL and HDL.

\begin{tabular}{|c|c|c|c|c|c|c|c|}
\hline & & \multicolumn{4}{|c|}{ Mean (SD) } & \multicolumn{2}{|c|}{ Dif. Groups } \\
\hline \multirow[t]{5}{*}{ Glycerol (mmol/l) } & & EG & & PG & & $p^{b} \mathrm{EG}$ & \\
\hline & & M0 & M1 & M0 & M1 & M1 & M1-M0 \\
\hline & & 0.04 & 0.15 & 0.04 & 0.09 & 0.003 & 0.004 \\
\hline & & $(0.04)$ & $(0.09)$ & $(0.02)$ & $(0.07)$ & & \\
\hline & $p^{a}$ M0 Vs M1 & 0.001 & & 0.001 & & & \\
\hline \multirow[t]{3}{*}{ Total Cholesterol (mg/dl) } & & 168.63 & 171.46 & 161.17 & 166.22 & 0.511 & 0.373 \\
\hline & & $(40.93)$ & $(40.41)$ & $(29.42)$ & $(31.23)$ & & \\
\hline & $p^{a}$ M0 Vs M1 & 0.174 & & 0.001 & & & \\
\hline \multirow[t]{3}{*}{ Triglycerides (mg/dl) } & & 88.58 & 87.67 & 84.07 & 90.05 & 0.791 & 0.078 \\
\hline & & $(38.53)$ & $(36.02)$ & $(45.70)$ & $(45.01)$ & & \\
\hline & $p^{a}$ M0 Vs M1 & 0.755 & & 0.023 & & & \\
\hline \multirow[t]{3}{*}{$\mathrm{LDL}(\mathrm{mg} / \mathrm{dl})$} & & 69.75 & 80.17 & 76.24 & 77.69 & 0.684 & 0.999 \\
\hline & & $(23.21)$ & $(21.05)$ & (16.01) & $(16.04)$ & & \\
\hline & $p^{a}$ M0 Vs M1 & 0.164 & & 0.005 & & & \\
\hline \multirow[t]{3}{*}{$\mathrm{HDL}(\mathrm{mg} / \mathrm{dl})$} & & 61.00 & 60.94 & 55.67 & 56.55 & 0.179 & 0.596 \\
\hline & & (6.09) & (10.51) & $(7.88)$ & $(8.96)$ & & \\
\hline & $p^{a}$ M0 Vs M1 & 0.797 & & 0.418 & & & \\
\hline
\end{tabular}

Abbreviations EG. experimental group; PG placebo group; M0, baseline moment; M1, after the intervention protocol; M1-M0. difference variable.

$p^{a}$ value with paired-samples $t$-test; $p^{b}$ value with independent $t$-test. 
Table 5

Correlation between glycerol mobilization (M1-M0). and abdominal fat. cholesterol. triglycerides and anthropometric measurements.

\begin{tabular}{|c|c|c|c|c|c|c|c|c|c|c|c|}
\hline & & $\begin{array}{l}\text { TG (mg/ } \\
\mathrm{dl})\end{array}$ & $\begin{array}{l}\text { CT (mg/ } \\
\mathrm{dl})\end{array}$ & $\begin{array}{l}\text { Total Fat } \\
(\%)\end{array}$ & $\begin{array}{l}\text { Waist Circum- } \\
\text { ference }(\mathrm{cm})\end{array}$ & $\begin{array}{l}\text { SAT- waist } \\
(\mathrm{mm})\end{array}$ & $\begin{array}{l}\text { VAT- waist } \\
(\mathrm{mm})\end{array}$ & $\begin{array}{l}\text { Abdomi-nal } \\
\text { Skinfold (mm) }\end{array}$ & $\begin{array}{l}\text { Supra-iliac } \\
\text { Skinfold (mm) }\end{array}$ & $\begin{array}{l}\mathrm{BMI}(\mathrm{Kg} / \\
\left.\mathrm{m}^{2}\right)\end{array}$ & $\begin{array}{l}\text { IPAQ (MET-min/ } \\
\text { week) }\end{array}$ \\
\hline $\begin{array}{l}\text { Glycerol } \\
\quad(\mathrm{mmol} / \mathrm{l})\end{array}$ & $\mathrm{EG}$ & $\begin{array}{l}r=-0.239 \\
p=0.133 \\
r=0.114 \\
p=0.471\end{array}$ & $\begin{array}{l}r=-0.193 \\
p=0.228 \\
r=0.260 \\
p=0.096\end{array}$ & $\begin{array}{l}\mathrm{r}=0.446 \\
\mathrm{p}=0.004 \\
\mathrm{r}=0.398 \\
\mathrm{p}=0.009\end{array}$ & $\begin{array}{l}r=0.353 \\
p=0.023 \\
r=-0.030 \\
p=0.849\end{array}$ & $\begin{array}{l}r=0.382 \\
p=0.015 \\
r=1.85 \\
p=0.266\end{array}$ & $\begin{array}{l}r=0.621 \\
p=0.002 \\
r=0.363 \\
p=0.105\end{array}$ & $\begin{array}{l}r=0.346 \\
p=0.027 \\
r=0.185 \\
p=0.241\end{array}$ & $\begin{array}{l}r=0.409 \\
p=0.008 \\
r=0.152 \\
p=0.336\end{array}$ & $\begin{array}{l}r=0.267 \\
p=0.092 \\
r=0.059 \\
p=0.711\end{array}$ & $\begin{array}{l}r=-0.086 \\
p=0.601 \\
r=0.027 \\
p=0.867\end{array}$ \\
\hline
\end{tabular}

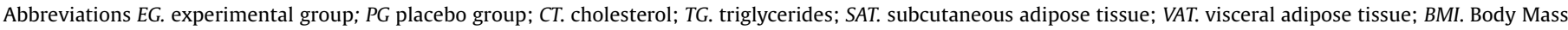
Index; IPAQ. international physical activity questionnaire.

$p$ value and $\mathrm{r}$ value from Pearson correlation.

of glycerol released and waist circumference, subcutaneous fat, visceral fat and abdominal and suprailiac skinfold. This suggests that exercise increases lipolysis preferably in individuals with greater abdominal fat mass, as previously described, which suggests that individuals with higher fat have higher metabolic activity due to higher lipolytic response and lower insulin sensitivity [47]. On the other hand, we did not observe these results in the experimental group, which showed a weak correlation, suggesting that the application of exercise associated with microcurrent may stimulate lipolysis in both high and low abdominal fat mass individuals.

We registered a positive correlation between total body fat and glycerol mobilization in both groups althought the application of microcurrent was local, abdominal region, suggesting it could due to the exercise effect.

Gender analysis showed significantly higher glycerol levels in the experimental group than in the placebo group after the intervention in both genders. Moreover, in the experimental group and placebo group females presented greater glycerol mobilization than males. After the intervention female glycerol mobilization was $140 \%$ and $60 \%$ compared to baseline level in EG and PG, respectively. The same profile was verified in male glycerol mobilization (90\% and 30\% compared to baseline level in EG and PG, respectively). In fact, other studies have shown greater glycerol mobilization in women during and after moderate aerobic exercise $[48,49]$. This gender-dependent mobilization is yet to be completely explained. Glisezinski et al. (2007) suggested this could be due to the higher quantity of adipose tissue in women [50]. Thus, higher fat mass may correlate to glycerol-measured lipolytic activity [51], enhanced by exercice in association with

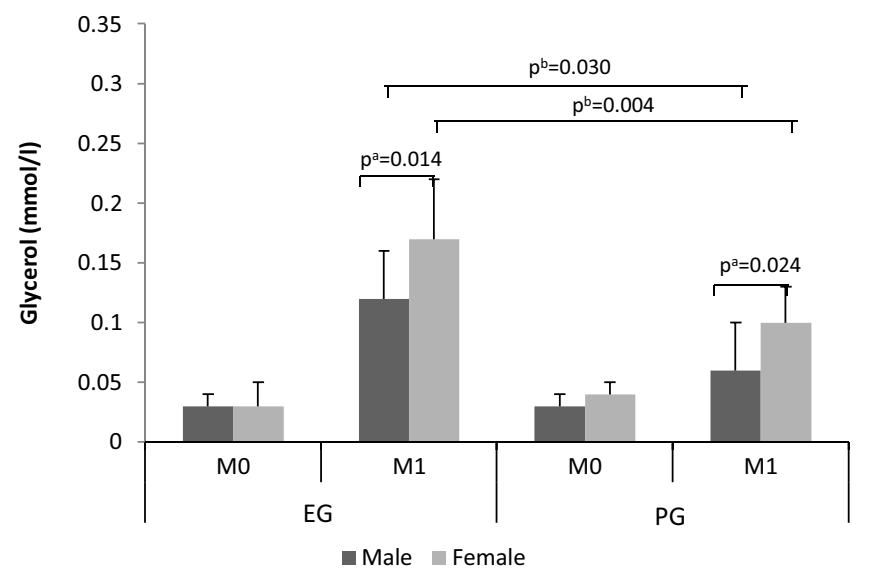

Fig. 2. - Glycerol mobilization at baseline and follow up, by gender.

Abbreviations: EG, experimental group; PG placebo group; MO, baseline moment; M1, after the intervention protocol; Data are expressed as median (Md) and Interquartile deviation (IQR) Pa value with Mann-Whitney Male vs female Pb value with Mann-Whitney GE vs GP. microcurrent. These diferences between genders may be related not only to adipocite's sensitivity, but also to specific lipolytic properties [52,53] and balance between $\beta$ - and $\alpha$-adrenergic receptors. In fact, the distribution of $\beta$ - and $\alpha$-adrenergic receptors in the abdominal region is gender-dependent, with women's adipocytes presenting lower number of $\alpha$-adrenergic receptors and lower sensitivity in the abdominal region [54,55]. Considering that these receptors inhibit lipolysis [56] and determine the lipolytic response [52], their lower activity in the abdominal area in women may be related to the results obtained in the present study, and suggests that these may be independent of gender differences in catecholamine concentrations during physical exercise [52].

Hormonal factors further explain these differences between genders, particularly estrogen concentration and the proportion of muscle fibers which interfere with the effects of physical exercise, and even microcurrent $[9,57,58]$. In fact, women have an increased number of oxidative muscle fibers (type I fibers) [59] that increase the concentration of HSL compared to muscles rich in type II fibers [34].

Some limitations of the present study should be addressed. On average, the subjects in our study showed most measured parameters near or at the recommended levels, which limits the generalization of our findings to high-riskc subjects. In order to complement this study, we suggest the identification and control of participants' basal metabolism through respiratory coefficient evaluation. Further studies should cover blood tests between the microcurrent application and the exercise protocol, in order to identify the exact amount of glycerol, triglycerides, total cholesterol, LDL and HDL released by microcurrent, and also over time (hours after protocol intervention) to see how long the effect lasts.

\section{Conclusion}

A single abdominal microcurrent session seems to have an additional effect to aerobic exercise, increasing glycerol mobilization to blood stream, due to an increase in the hydrolysis of triglycerides.

Males and females benefit from the application of microcurrent with exercise, experiencing an increase in lipolytic activity.

The application of microcurrent is advantageous in individuals with different levels of abdominal fat.

\section{Conflict of interest}

The authors have no conflicts of interest to declare. All authors have reviewed the manuscript and take responsibility for its content.

\section{Acknowledgments}

The authors are grateful to the participants and Nuno Duarte for the English Review. 


\section{References}

[1] A. Sironi, et al., Impact of increased visceral and cardiac fat on cardiometabolic risk and disease, Diabet. Med. 29 (5) (2012) 622-627.

[2] K. Kishida, et al., Visceral adiposity as a target for the management of the metabolic syndrome, Ann. Med. 44 (3) (2012) 233-241.

[3] I.J. Neeland, et al., Dysfunctional adiposity and the risk of prediabetes and type 2 diabetes in obese adults, JAMA 308 (11) (2012) 1150-1159.

[4] A. Tchernof, et al., Regional differences in adipose tissue metabolism in women: minor effect of obesity and body fat distribution, Diabetes 55 (5) (2006) 1353-1360.

[5] K. Marinou, et al., Structural and functional properties of deep abdominal subcutaneous adipose tissue explain its association with insulin resistance and cardiovascular risk in men, Diabetes Care 37 (3) (2014) 821-829.

[6] A. Chandra, et al., The relationship of body mass and fat distribution with incident hypertension: observations from the Dallas heart study, J. Am. Coll. Cardiol. 64 (10) (2014) 997-1002.

[7] M. Lafontan, D. Langin, Lipolysis and lipid mobilization in human adipose tissue, Prog. Lipid Res. 48 (5) (2009) 275-297.

[8] T. Scheers, et al., Lipid profile in men and women with different levels of sports participation and physical activity, Public Health Nutr. 11 (11) (2008) 1098-1106.

[9] T. Oosthuyse, A.N. Bosch, Oestrogen's regulation of fat metabolism during exercise and gender specific effects, Curr. Opin. Pharmacol. 12 (3) (2012) 363371.

[10] J.F. Trejo-Gutierrez, G. Fletcher, Impact of exercise on blood lipids and lipoproteins, J. Clin. Lipidol. 1 (3) (2007) 175-181.

[11] S.E. Chiuve, et al., Alternative dietary indices both strongly predict risk of chronic disease, J. Nutr. (2012) (p. jn. 111.157222).

[12] X. Cheneviere, et al., Effect of a 1-hour single bout of moderate-intensity exercise on fat oxidation kinetics, Metabolism 58 (12) (2009) 1778-1786.

[13] J.F. Horowitz, S. Klein, Lipid Metabolism During Endurance Exercise, American Society for Clinical Nutrition, 2000 72(0002-9165 (Print)): p. 6.

[14] M. Ahmadian, Y. Wang, H.S. Sul, Lipolysis in adipocytes, Int. J. Biochem. Cell Biol. 42 (5) (2010) 555-559.

[15] B.K. Pedersen, M.A. Febbraio, Muscles, exercise and obesity: skeletal muscle as a secretory organ, Nat. Rev. Endocrinol. 8 (8) (2012) 457-465.

[16] A. Noites, et al., Effects of aerobic exercise associated with abdominal microcurrent: a preliminary study, J. Altern. Complement. Med. 21 (4) (2015) 229-236.

[17] Z.H. Hamida, et al., Effect of electrical stimulation on lipolysis of human white adipocytes, Appl. Physiol. Nutr. Metab. 36 (2) (2011) 271-275.

[18] K. Melzer, Carbohydrate and fat utilization during rest and physical activity, eSPEN J. 6 (2) (2011) e45-e52.

[19] K. Frayn, Fat as a fuel: emerging understanding of the adipose tissue-skeletal muscle axis, Acta Physiol. 199 (4) (2010) 509-518.

[20] E.E. Blaak, W.H. Saris, Substrate oxidation, obesity and exercise training, Best Pract. Res. Clin. Endocrinol. Metab. 16 (4) (2002) 667-678.

[21] L.J. van Loon, et al., The effects of increasing exercise intensity on muscle fuel utilisation in humans, J. Physiol. 536 (1) (2001) 295-304.

[22] U. Kyle, et al., Bioelectrical impedance analysis-part II: utilization in clinical practice, Clin. Nutr. 23 (2004) 1430-1453.

[23] W.H. Organization, Physical Status: The Use and Interpretation of Anthropometry, 86, 1995

[24] R. Eston, T. Reilly, Kinantrhopometry and Exercise Physiology Laboratory Manual, in Tests, Procedures and Data, Routledge : 2 Park Square, Milton Park, Abingdon, Oxon, 2009.

[25] C.L. Craig, et al., International physical activity questionnaire: 12-country reliability and validity, Med. Sci. Sports Exerc. 35 (8) (2003) 1381-1395.

[26] Marshall A. and A. Bauman The International Physical Activity Questionnaire: Summary Report of the Reliability \& Validity Studies 2001. 25.

[27] C.et al. Lopes, Intake and adipose tissue composition of fatty acids and risk of myocardial infarction in a male Portuguese community sample, J. Am. Diet. Assoc. 107 (2) (2007) 10.

[28] A.S.C. Melo, et al., Clay body wrap with microcurrent: effects in central adiposity, Appl. Clay Sci. 80-81 (2013) 6.

[29] A. Noites, et al., Effects of microcurrents and physical exercise on the abdominal fat in patients with coronary artery disease, EuJIM 7 (5) (2015) 499507.

[30] ACSM's Guidelines for Exercise Testing and Prescription. 2010, Canada: Wolters Kluwer | Lippincott Wiliams \& Wilkins.

[31] H. Tanaka, K.D. Monahan, D.R. Seals, Age-predicted maximal heart rate revisited, J. Am. Coll. Cardiol. 37 (1) (2001) 153-156.
[32] J. Achten, A.E. Jeukendrup, Optimizing fat oxidation through exercise and diet, Nutrition 20 (7-8) (2004) 716-727.

[33] A. Jeukendrup, Modulation of carbohydrate and fat utilization by diet: exercise and environment, Biochem. Soc. Trans. 31 (6) (2003) 1270-1273.

[34] J.W.E. Jocken, et al., Hormone-sensitive lipase serine phosphorylation and glycerol exchange across skeletal muscle in lean and obese subjects: effect of B-Adrenergic stimulation, Diabetes 57 (7) (2008) 1834-1841.

[35] J.F. Horowitz, Fatty acid mobilization from adipose tissue during exercise, Trends Endocrinol. Metab. 14 (8) (2003) 386-392.

[36] M.P. Ramirez-Ponce, et al., Noradrenaline modulates the electrical activity of white adipocytes by a cAMP-dependent mechanism, J. Endocrinol. 159 (3) (1998) 397-402.

[37] M.P. Ramirez-Ponce, J.C. Mateos, J.A. Bellido, Insulin increases the density of potassium channels in white adipocytes: possible role in adipogenesis, J. Endocrinol. 174 (2) (2002) 299-307.

[38] M.P. Ramírez-Ponce, J.C. Mateos, J.A. Bellido, Human adipose cells have voltage-dependent potassium currents, J. Membr. Biol. 196 (2) (2003) 129134.

[39] T. Kondo, et al., Mild electrical stimulation with heat shock reduces visceral adiposity and improves metabolic abnormalities in subjects with metabolic syndrome or type 2 diabetes: randomized crossover trials, EBioMedicine 1 (1) (2014) 80-89.

[40] V.E. Chaves, D. Frasson, N.H. Kawashita, Several agents and pathways regulate lipolysis in adipocytes, Biochimie 93 (10) (2011) 1631-1640.

[41] C. Moro, et al., Exercise-induced lipid mobilization in subcutaneous adipose tissue is mainly related to natriuretic peptides in overweight men, Am. J Physiol.-Endocrinol. Metab. 295 (2) (2008) E505-E513.

[42] A.B. Jordy, B. Kiens, Regulation of exercise-induced lipid metabolism in skeletal muscle, Exp. Physiol. 99 (12) (2014) 1586-1592.

[43] S.K. Powers, E.T. Howley, Exercise Physiology: Theory and Application to Fitness and Performance, MCGRAW-HILL Higher Education., 2012.

[44] M. Ahmadian, R.E. Duncan, H.S. Sul, The skinny on fat: lipolysis and fatty acid utilization in adipocytes, Trends Endocrinol. Metab. 20 (9) (2009) 424-428.

[45] M.C. Venables, J. Achten, A.E. Jeukendrup, Determinants of fat oxidation during exercise in healthy men and women: a cross-sectional study, J. Appl. Physiol. (1985) 98 (1) (2005) 160-167.

[46] R.P. Hernandez-Torres, et al., Effects of single sessions of low-intensity continuous and moderate-intensity intermittent exercise on blood lipids in the same endurance runners, J. Sci. Med. Sport 12 (2) (2009) 323-331.

[47] M.M. Ibrahim, Subcutaneous and Visceral Adipose Tissue: Structural and Functional Differences, Oxford, UK, 2010, pp. 11-18.

[48] S.L. Carter, C. Rennie, M.A. Tarnopolsky, Substrate utilization during endurance exercise in men and women after endurance training, Am. J. Physiol.: Endocrinol. Metab. 280 (2001) 898-907.

[49] C.A. Vella, L. Kravitz, Gender Differences in Fat Metabolism, in Idea Health \& Fitness Source, San Diego, California, 2002, pp. 3-4.

[50] I.d. Glisezinski, Mobilisation des lipides du tissu adipeux au cours de l'exercice physique, Sci. Sports (2007) 280-285.

[51] G.C. Henderson, et al., Plasma triglyceride concentrations are rapidly reduced following individual bouts of endurance exercise in women, Eur. J. Appl. Physiol. 109 (4) (2010) 721-730.

[52] B. Mittendorfer, J.F. Horowitz, S. Klein, Effect of gender on lipid kinetics during endurance exercise of moderate intensity in untrained subjects, Am. J. Physiol.-Endocrinol. Metab. 2002 (2016) 58-65.

[53] T.J. Horton, et al., Greater systemic lipolysis in women compared with men during moderate-dose infusion of epinephrine and/or norepinephrine, J. Appl. Physiol. 107 (1) (2009) 200-210.

[54] J. Bulow, et al., Lipid mobilization from human abdominal, subcutaneous adipose tissue is independent of sex during steady-state exercise, Clin. Physiol. Funct. Imaging 2006 (2016) 205-211.

[55] E. Blaak, Gender differences in fat metabolism, Curr. Opin. Clin. Nutr. Metab. Care 4 (2001) 499-502.

[56] P. Imbeault, et al., Reduced $\alpha 2$-adrenergic sensitivity of subcutaneous abdominal adipocytes as a modulator of fasting and postprandial triglyceride levels in men, J. Lipid Res. 41 (9) (2000) 1367-1375.

[57] M.A. Tarnopolsky, Gender differences in substrate metabolism during endurance exercise, Can. J. Appl. Physiol. 25 (4) (2000) 312-327.

[58] M.A. Tarnopolsky, Sex differences in exercise metabolism and the role of 17beta estradiol, Med. Sci. Sports Exerc. 40 (4) (2008) 648-654.

[59] B. Norman, et al., Strength: power, fiber types, and mRNA expression in trained men and women with different ACTN3 R577X genotypes, J. Appl. Physiol. 106 (3) (2009) 959-965. 